\title{
SINGLE PROLONGED STRESS: TOWARD AN ANIMAL MODEL OF POSTTRAUMATIC STRESS DISORDER
}

\author{
Shigeto Yamamoto, M.D. Ph.D., ${ }^{1}$ Shigeru Morinobu, M.D. Ph.D.., ${ }^{1,3}$ Shiro Takei, M.D.., ${ }^{1}$ Manabu Fuchikami, M.D. Ph.D., ${ }^{1}$
} Aya Matsuki, ${ }^{1}$ Shigeto Yamawaki, M.D. Ph.D. ${ }^{1,3}$ and Israel Liberzon, M.D. ${ }^{2}$

\begin{abstract}
Although selective serotonin reuptake inhibitors (SSRIs) are reported to be effective in decreasing posttraumatic stress disorder (PTSD) symptoms, a subgroup of PTSD patients remain chronically symptomatic and maintain conditioned fear responses to traumatic stimuli. In this context, the establishment of an appropriate animal model of PTSD is necessary to promote better understanding of the mechanisms of the disorder and to facilitate the development of more effective therapeutic alternatives to SSRIs. Although no single widely accepted animal model of PTSD has been established to date, the single prolonged stress (SPS) animal model bas been partially validated as a model for PTSD. SPS rats mimic the pathophysiological abnormalities and behavioral characteristics of PTSD, such as enhanced anxiety-like bebavior and glucocorticoid negative feedback, and they exhibit the expected therapeutic response to paroxetine on enbanced fear memory. In addition, SPS rats exhibit enhanced freezing in response to contextual fear conditioning, and impaired extinction of fear memory, which is alleviated by D-cycloserine. The enhanced consolidation and impaired extinction of fear memory found in SPS rats suggests that this model has additional value because recent studies of PTSD indicate that memory abnormalities are a central feature. In this study, we summarize the behavioral and pathophysiological PTSDlike symptoms in SPS, focusing on memory abnormalities, and evaluate the validity of SPS as an animal model of PTSD. Depression and Anxiety 26:1110-1117, 2009. (c) 2009 Wiley-Liss, Inc.
\end{abstract}

Key words: posttraumatic stress disorder; single prolonged stress; animal model; contextual fear; fear extinction

\begin{abstract}
'Department of Psychiatry and Neurosciences, Graduate School of Biomedical Sciences, Hiroshima University, Hiroshima, Japan ${ }^{2}$ Department of Psychiatry, University of Michigan, Ann Arbor, Michigan

${ }^{3}$ Project of Core Research for Evolutional Science and Technology (CREST) of Japan Science and Technology
\end{abstract}

Contract grant sponsors: Ministry of Education, Science, and Culture of Japan; Ministry of Health and Welfare of Japan; Japan Science and Technology (JST). Supported by VA Merit Award and CDMPR DOD Investigator Initiated Research Award, to IL.

*Correspondence to: Shigeru Morinobu, Department of Psychiatry and Neurosciences, Division of Frontier Medical Science, Programs for Biomedical Research, Graduate School of Biomedical Sciences, Hiroshima University, 1-2-3 Kasumi, Minami-ku, Hiroshima 734-8551, Japan. E-mail: smoriob@ hiroshima-u.ac.jp

\section{INTRODUCTION}

$\mathbf{P}$ Ptress disorder (PTSD) develops according to the DSM-IV diagnostic criteria, involves characteristic features such as persistent experiencing of trauma, avoidance, numbing, and hyperarousal. ${ }^{[1]}$ Although many individuals with PTSD recover during the first couple of years following traumatic exposure, up to $30-40 \%$ remain chronically symptomatic. ${ }^{[2]}$ Furthermore, individuals with chronic PTSD were shown to maintain conditioned fear responses to traumatic stimuli

Received for publication 23 July 2009; Revised 15 September 2009; Accepted 15 September 2009

DOI 10.1002/da.20629

Published online 16 November 2009 in Wiley InterScience (www. interscience.wiley.com).

(C) 2009 Wiley-Liss, Inc. 
even $40-50$ years after the trauma. ${ }^{[3]}$ Thus, the high prevalence, chronicity, and resistance to treatment underscore the importance of the development of effective therapeutic strategies for PTSD. In this context, the establishment of an appropriate animal model of PTSD can promote our understanding of the mechanisms of PTSD and may help to identify novel and more effective therapeutic strategies.

Ultimately, the optimal animal model would mimic the pathophysiological abnormalities and behavioral characteristics of PTSD and involve exposure to trauma-like events. No single widely accepted animal model of PTSD has been established to date and there is an ongoing debate over what constitutes a valid animal model for this disorder. Among various animal models of PTSD proposed, a single prolonged stress (SPS) model, proposed by Liberzon et al., replicates the specific neuroendocrinological abnormalities observed in PTSD patients ${ }^{[4,5]}$ such as enhanced glucocorticoid negative feedback. According to their method, SPS is conducted in three stages. Briefly, animals are restrained for $2 \mathrm{hr}$ and immediately afterwards undergo a $20 \mathrm{~min}$ forced swim in $24^{\circ} \mathrm{C}$ water. The forced swim is performed with six rats at a time in an 18 gallon plastic tub $(55.6 \mathrm{~cm}$ diameter, $45.4 \mathrm{~cm}$ height) two-thirds full. Following recuperation for $15 \mathrm{~min}$, animals are exposed to ether until the loss of consciousness and then left undisturbed in their home cage for 7 days. Consistent with time-dependent sensitization (TDS) studies, ${ }^{[6]}$ it was proposed that the undisturbed period is a necessary condition to produce PTSD-like manifestations. As a result, behavioral experiments are generally undertaken 7-14 days after the SPS procedure.

SPS is not the only animal model of PTSD that has been proposed, and various animal paradigms have been developed in an attempt to model the disorder. These include exposure to various stressors such as electric shock, ${ }^{[7-11]}$ underwater trauma, ${ }^{[12]}$ and exposure to predators $^{[13,14]}$ or predator-related cues. ${ }^{[12,15,16]}$ Exposure to these stressors leads to an increase in anxiety-like behaviors, and in some cases, exaggerated startle responses, cognitive impairment, enhanced fear conditioning, and reduced social interaction. ${ }^{[17]}$ Some studies have also reported physiological changes (e.g. hypothalamo-pituitary-adrenal (HPA) axis) resembling those observed in patients with PTSD. ${ }^{[1,18]}$ Studies using inescapable shock (IS) in a shuttle-box are of particular interest. Rats exposed to IS exhibit PTSD-like bidirectional behavioral changes; that is, "avoidance/ numbing" (e.g. decreased activity, reactivity, and interest in surroundings) and "hyperarousal" (e.g. irritability and exaggerated responsiveness to external stimuli) in an avoidance/escape task session ( 2 weeks after IS). ${ }^{[7,19]}$

Each of these animal models has some degree of face and construct validity for PTSD; however, it is beyond the scope of the present work to provide a detailed comparison of these animal models. In this article, we describe the PTSD-like behavioral and pathophysiological symptoms of SPS and discuss the validity of SPS as an animal model of PTSD.

\section{PTSD-LIKE SYMPTOMS IN SPS HYPOTHALAMO-PITUITARY-ADRENAL (HPA) AXIS}

The biological basis of PTSD is still largely unknown. However, various HPA axis abnormalities have been repeatedly reported in PTSD, including low levels of cortisol in urine and plasma, enhanced suppression of cortisol in response to administration of low-dose dexamethasone (a synthetic corticosteroid), and glucocorticoid receptor abnormalities. ${ }^{[20,21]} \mathrm{Ex}-$ aggerated suppression of plasma cortisol in response to low-dose dexamethasone appears to be the most commonly reported finding. ${ }^{[22]}$ Yehuda et al. examined the levels of cortisol and the number of lymphocyte glucocorticoid receptors before and after administration of 0.5 or $0.25 \mathrm{mg}$ dexamethasone in 14 combat veterans with PTSD, 12 combat veterans without PTSD, and 14 nonpsychiatric healthy men. At both doses of dexamethasone, combat veterans with PTSD showed greater suppression of cortisol as compared with combat veterans without PTSD and normal controls. ${ }^{[23]}$ These clinical findings suggest that an appropriate animal model of PTSD would exhibit enhanced negative feedback of the HPA axis after exposure to synthetic glucocorticoids.

Liberzon et al. originally developed the SPS model and found that it was associated with enhanced negative feedback of the HPA axis, as indicated by plasma ACTH levels after cortisol administration. ${ }^{[4]}$ Furthermore, to determine the neural mechanisms of enhanced negative feedback of the HPA axis, Liberzon et al. also examined regional changes in Type I (MR) and Type II (GR) glucocorticoid receptor mRNA distribution in the hippocampus using in situ hybridization. ${ }^{[5]}$ Five group of rats were studied: control group, SPS -7 days group (no sensitization period), SPS group, SPS +7 days group (14 days sensitization), and SPS+chronic stress group (chronic stress during 7 days sensitization). The SPS + chronic stress group was exposed to four different stressors on a variable schedule for 7 days. Twenty-four hours after SPS, down-regulation of GR and MR mRNA was found across all hippocampal subfields. Seven days later (full SPS group), there was a differential recovery, with GR mRNA reaching higher than the prestress levels and MR mRNA remaining downregulated. The same differential regulation was present in the SPS +7 days group. The SPS + chronic stress group, which exhibited normally fast feedback, also showed normalization of GR and MR mRNA levels. It was concluded that a sensitization period of 7 days was necessary (SPS-7, and SPS + chronic stress controls), which led to persistent changes (SPS +7 days) in the glucocorticoid receptor ratio in hippocampus. As hippocampal glucocorticoid receptors play a central role in glucocorticoid negative feedback regulation, ${ }^{[24]}$ these findings suggest that the increase in GR in the hippocampus is involved in the fast feedback hypersensitivity observed in SPS rats. 


\section{GLUTAMATE AND GABA}

Although HPA abnormalities are probably the most specific neurobiological findings associated with PTSD, the complex symptoms associated with the disease suggest involvement of other neurotransmitters and various circuits governing cognitive and emotional processing. Reliable animal models provide the opportunity to examine these candidate systems such as the major excitatory (glutamatergic) and inhibitory (Gammaaminobutyric acid (GABA)) neurotransmitter systems. Dysfunction in brain glutamatergic systems, involving $N$-methyl-D-aspartate (NMDA) receptors in particular, has been suggested as a neurobiological component of PTSD, perhaps contributing to hippocampal toxicity. ${ }^{[25]}$ Harvey et al. demonstrated in a radioligand binding assay that NMDA receptor density was reduced in the rat hippocampus after SPS. ${ }^{[26]}$ In concert with the change in NMDA receptor density, spatial memory deficits in the Morris water maze were exhibited. ${ }^{[6]}$ As NMDA pathways in the hippocampus are crucial for memory function, ${ }^{[27]}$ the reduction in NMDA receptor density in this study may underlie the cognitive changes observed in the SPS model. ${ }^{[6]}$ We also have observed NMDA changes in SPS rats, such as significant upregulation of hippocampal NMDA receptor subunit mRNAs 7 days after SPS. ${ }^{28]}$ Although the reported changes in receptor density and subunit mRNA appear to occur in opposition to one another, the precise mechanism by which receptor density and subunit changes translate into functional deficits remain unknown; it would therefore be premature to consider them contradictory. It is also plausible that the difference in experimental procedures (e.g. modified SPS vs. SPS, duration after exposure to SPS) contributed to this discrepancy. Together these findings suggest, however, that glutamatergic-NMDA systems might be altered in SPS animals.

GABA(A) receptors are thought to play an important role in modulating the central nervous system in response to stress. ${ }^{[29]}$ Geuze et al. reported differences in the benzodiazepine-GABA(A) receptor complex in PTSD using [(11)C]-flumazenil and positron emission tomography. ${ }^{[30]}$ PTSD veterans showed significantly reduced [(11)C]-flumazenil binding throughout the cortex, hippocampus, and thalamus. Harvey et al. reported reduced hippocampal GABA levels, as measured by high-performance liquid chromatography, in a rat model of SPS. ${ }^{[26]}$ Furthermore, Khan and Liberzon demonstrated that topiramate (which has GABA(A)modulating properties) attenuated the enhanced acoustic startle response in SPS rats. ${ }^{[31]}$ Together, these findings support the hypothesis that hypofunction of the GABAergic pathway could be, at least in part, involved in the pathophysiology of PTSD.

\section{FEAR RESPONSE}

Trauma-related and -unrelated fear response. In addition to their value for studying neurotransmitters, animal models are useful for examining specific brain circuitry that might be involved in PTSD pathophysiology. In this respect, SPS animals have been used to examine fear response circuitry implicated in PTSD, since some PTSD symptoms, like exaggerated reaction to traumarelated events and re-experiencing, suggest that fear learning or fear conditioning are involved. Patients with PTSD also demonstrate hyperarousal and hypervigilance responses to more general aversive (trauma-unrelated) stimuli. ${ }^{[32,33]}$ Enhanced autonomic arousal responses, such as increased skin conductance, have been reported in PTSD, and are induced by stressors unrelated to trauma. ${ }^{[33]}$ Orr et al. reported larger heart rate responses to sudden, loud tones (trauma-unrelated) in Vietnam combat veterans with PTSD. ${ }^{[32]}$

Similarly, a number of animal studies have reported that SPS rats exhibit enhanced trauma-unrelated fear. ${ }^{[34-37]}$ In these studies, rats were first subjected to SPS and then exposed to contextual fear conditioning, where fear-inducing stimuli and the context are not associated with initial trauma (SPS). In a study by Takahashi et al., ${ }^{[36]}$ the contextual fear paradigm was performed after a 14-day undisturbed period following SPS. On the first day of fear conditioning, rats were exposed to the context $(180 \mathrm{~s}$, in the conditioning chamber $(325 \mathrm{~W} \times 280 \mathrm{H} \times 500 \mathrm{Dmm})$ without any stimulation). Immediately after that, they were administered a foot shock $(0.8 \mathrm{~mA}, 4 \mathrm{~s})$. Twenty-four hours later, the rats were placed back in the conditioning chamber where the foot shock was delivered, and the duration of freezing was evaluated. SPS rats showed a significant increase in contextual freezing as compared with rats not subjected to SPS.

In addition, as a method of evaluating the nonassociative fear response, symptoms of hypervigilance and hyperarousal can be assessed by measuring increases in the amplitude of startle responses to tones or airpuffs of defined intensity. In a study by Khan and Liberzon, SPS rats were found to exhibit increased startle responses to $50 \mathrm{~ms}$ and $108 \mathrm{~dB}$ tones, both when compared with a nonstressed control group or with their own startle responses before the SPS session. ${ }^{[31]}$

To our knowledge, no studies reported in the literature have examined fear responses to traumarelated stimuli using the SPS paradigm. We, therefore, examined behavioral activity in response to the forced swimming test (FST) to evaluate trauma-related fear in SPS rats. The experimental design is shown in Figure 1. The test was conducted 7 days after the SPS procedure. In the control group, rats first underwent forced swimming for $20 \mathrm{~min}$, instead of the SPS procedure. In both groups, immobility was measured in comparison with the first FST session (for the first $5 \mathrm{~min}$ of the $20 \mathrm{~min}$ period). The results showed that the duration of immobility during the second FST was significantly longer than that during the first FST in the SPS group (Fig. 2). In contrast, there was no significant difference in immobility between the first and second FST in the sham group (Fig. 2). 


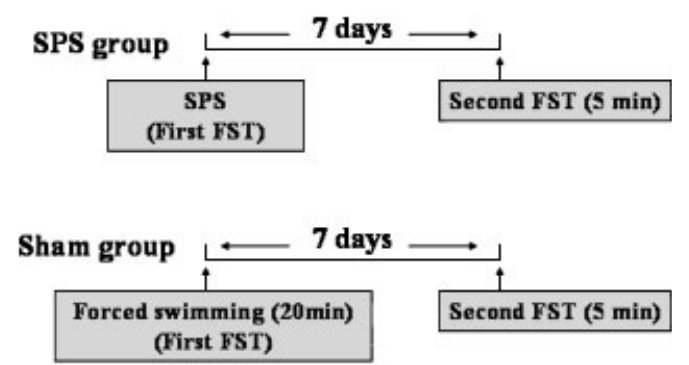

Figure 1. Treatment groups and procedure in the forced swimming test.

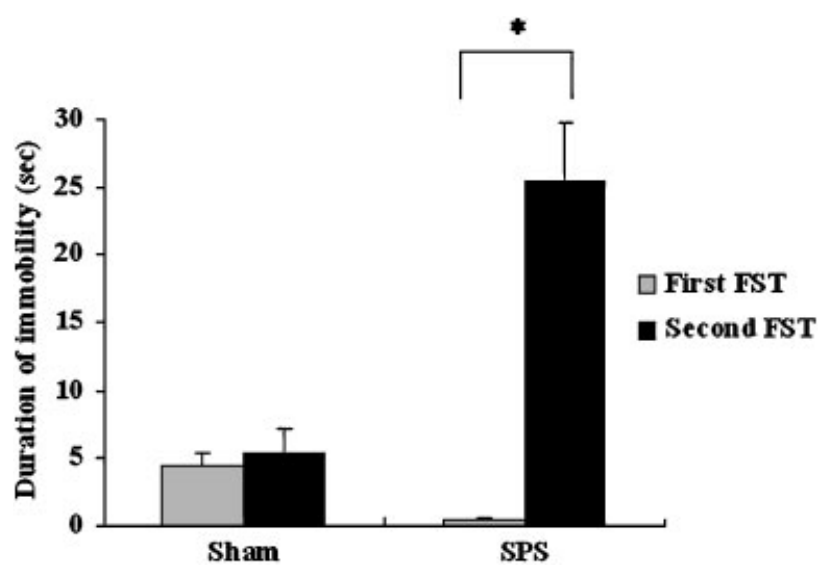

Figure 2. Comparison of immobility in the forced swimming test. Data are expressed as mean \pm SEM of 10 rats per group. The duration of immobility during the second FST was significantly longer than that during the first FST in the SPS group $[t(18)=5.91, P<0.05]$. There was no significant difference in immobility between the first and second FST in the sham group $[t(18)=0.38, P=0.71] .{ }^{*} P<0.05$; Unpaired Student's $t$-test.

These findings should be interpreted discreetly because there is a possibility that increased immobility represents not only the enhanced fear response in PTSD-like symptoms, but also the despair-like response in depression. Therefore, we performed the tail suspension test to differentiate the enhanced fear response from the despair-like response. The test was conducted 7 days after the SPS procedure. Rats were suspended by their tails for $6 \mathrm{~min}$, and the duration of immobility was measured according to the method described by Steru et al. ${ }^{[38,39]}$ The result showed that there was no significant difference in immobility between the SPS and sham groups, suggesting that SPS rats did not exhibit despair-like responses in this test (Fig. 3). Clinically, patients with PTSD often present with despair/helplessness in response to a stressful event, and in fact, the DSM-IV diagnostic criteria stipulate that the person's response to the event involves helplessness, besides intense fear or horror. ${ }^{[1]}$ Although it is difficult to discriminate the enhanced fear

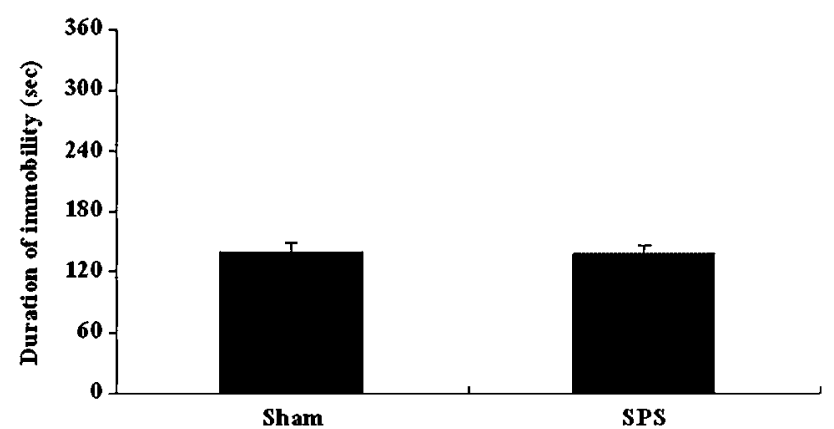

Figure 3. Comparison of immobility in the tail suspension test. Data are expressed as mean \pm SEM of nine rats per group. There was no significant difference in immobility between the SPS and sham groups according to unpaired Student's $t$-test $[t(16)=0.11, P=0.91]$.

response from the despair-like response based on these clinical findings, our results indicate that prolonged immobility in the second FST in SPS rats would reflect the fear response, rather than the despair-like response.

Fear extinction. PTSD patients exhibit long-lasting reexperience of traumatic events and avoidance of the trauma-related stimuli, even though they recognize that the traumatic event is no longer occurring. Recent advances in our understanding of the mechanisms underlying fear extinction have led to the hypothesis that dysfunctional fear extinction plays an important role in the development of clinical symptoms, such as reexperiencing of trauma, in PTSD. ${ }^{[40-43]}$ In fact, a recent study, in which a 2-day fear conditioning and extinction procedure was used, showed that PTSD patients are deficient in extinction recall. ${ }^{[44]}$ On day 1 of the study, subjects viewed colored light-conditioned stimuli, some of which were paired with mild electric shock, followed by extinction of the conditioned responses in another context. On the second day, extinction recall was tested in the previous extinction context. Skin conductance response was the dependent measure. The retention of extinction measured on the second day was deficient in the PTSD combat veterans relative to non-PTSD combat veterans.

In parallel, using animal models, we have recently examined the ability of SPS to impair fear extinction and to test whether D-cycloserine (DCS) can alleviate impaired fear extinction in SPS rats. ${ }^{[28]}$ In that study, contextual fear conditioning was conducted 7 days after the SPS procedure. Twenty-four hours after fear conditioning, rats were placed for $10 \mathrm{~min}$ without footshock in the same chamber where the footshock was first delivered. In a similar manner, extinction training was performed on each of 5 consecutive days following fear conditioning. DCS was administered daily for 6 days (from the end of fear conditioning to the beginning of the fifth extinction training session). SPS rats exhibited impaired fear extinction when compared with sham rats, and DCS ameliorated the impairment of fear extinction. 
In a human clinical pilot study, Heresco-Levy et al. demonstrated insufficient efficacy of DCS in the treatment of PTSD. ${ }^{[45]}$ In that study, DCS was given chronically without exposure therapy. More recent studies in animals and humans have showed that DCS enhances fear extinction when given in conjunction with extinction training. ${ }^{[46-49]}$ Therefore, it is important to note that DCS administration has only minimal effect unless it is combined with extinction training. Together, these findings support the notion that impaired extinction is the mechanism responsible for the development of PTSD re-experiencing, and that SPS animals mimic some of the same abnormalities in fear extinction seen in PTSD patients. Additional studies investigating increased resistance to extinction of the conditioned fear response are needed to further elucidate the validity of SPS as an animal model of PTSD

\section{OTHER PTSD-LIKE SYMPTOMS}

In addition to exaggerated fear responses, traumatic memories, and avoidance, PTSD has been associated with additional deficits/symptoms. Verbal memory impairments, indicative of hippocampal dysfunction, have been often reported in PTSD. ${ }^{[50-52]}$ Bremner et al. used a neuropsychological test as a probe of hippocampal function in 26 Vietnam veterans with combat-related PTSD and 15 matched healthy control subjects. ${ }^{[50]}$ Compared with controls, the combat veterans showed a decrease in immediate and delayed recall, as well as in percent retention, in the presence of similar IQ scores. The same group also reported similar results with adult survivors of severe childhood physical or sexual abuse who presented for psychiatric treatment. ${ }^{[51]}$

Using a modified SPS paradigm, Harvey et al. showed that these rats exhibit spatial memory deficits in the Morris water maze, ${ }^{[6]}$ and this finding was confirmed by Kohda et al. ${ }^{[35]}$ However, it is also possible that conditioned fear contributed to these learning deficits, as the original SPS session included a swim period. Richter-Levin evaluated the conditioning effects of an underwater trauma on the Morris water maze performance. ${ }^{[53]}$ Both $1 \mathrm{hr}$ and 3 weeks after the trauma, significant behavioral deficits were observed in the water maze; however, they were context specific. Underwater trauma in a different (out-of-context) water container had no effects on a spatial memory task in the water maze. Based on these findings, the SPS learning deficits in the water maze are not likely to be due to the effects of conditioned fear because the context of the water maze differs from that of the SPS session.

The clinical picture of PTSD often involves social isolation and discomfort in novel environments. These factors may be modeled in animal studies employing exploratory-based approach-avoidance conflict tests, which assess the impact of fear of open and/or brightly lit areas on the exploration of novel environments. Imanaka et al. reported that SPS rats showed a significant decrease in the percentage of open arm entries and the percentage of open arm time in the elevated plus maze test, indicating that SPS induces alterations of anxiety-related behavior. ${ }^{[37]}$

Finally, two studies ${ }^{[36,37]}$ examined stress-induced analgesia, which is often seen in PTSD patients ${ }^{[5]}$ and SPS animals. In the model used in these studies, animals are placed individually into the testing chamber and a flinch-jump test is performed while shocks are delivered to the grid floor of the chamber through a shock generator. After a 3-min period of habituation, upwards shock titrations are continued in a stepwise manner $(0.05 \mathrm{~mA}, 0.05-0.8 \mathrm{~mA}$ range). The flinch threshold is defined as the lowest shock intensity that elicited any detectable response. The vocalization threshold is defined as the lowest shock intensity that elicited vocalization, and the jump threshold, as the lowest shock intensity that elicited simultaneous removal of at least three paws (including both hindpaws) from the grid. In these studies, shock-induced vocalization and jump thresholds were significantly increased in SPS rats as compared with control animals.

\section{SPS AS AN ANIMAL MODEL OF PTSD}

The validity of an animal model is typically assessed using three validity criteria: phenomenological similarity (face validity), corresponding theoretical explanatory frameworks (construct validity), and the ability to predict that a pharmacological agent with efficacy demonstrated in animal studies to have a subsequent therapeutic effect in humans (predictive validity). ${ }^{[5]}$ The substantial evidence supporting the face and construct validity of the SPS model suggests that it could be suitable for the study of PTSD; however, there are unresolved issues that require further exploration. Regarding face validity, although there is accumulating evidence that SPS rats exhibit symptoms of increased arousal, such as exaggerated fear responses to traumarelated and -unrelated stimuli, avoidance of stimuli associated with trauma or emotional blunting have not been studied in SPS. With respect to construct validity, several SPS studies suggested hippocampal abnormality, which has also been implicated in patients with PTSD, but other brain areas such as the amygdala or medial prefrontal regions, implicated in PTSD by functional neuroimaging studies, ${ }^{[56]}$ have not been yet been investigated in the SPS model.

Although pharmacotherapy for PTSD is still at a relatively early stage of development, sustained administration of selective serotonin reuptake inhibitors (SSRIs) has proven to be highly effective in decreasing PTSD symptoms. ${ }^{[57]}$ Thus, the appropriate predictive ability can be demonstrated by chronic administration of SSRIs in the SPS model, which would be expected to ameliorate PTSD-like symptoms. In fact, several recent studies have demonstrated the efficacy of SSRIs on PTSD-like symptoms in SPS rats. ${ }^{[36,58,59]}$ Takahashi 
et al. showed that 14-day oral administration of paroxetine (PRX) immediately after exposure to SPS, at a dose sufficient to produce clinically relevant serum concentrations, was effective in the prevention of enhanced contextual freezing in SPS rats. ${ }^{[36]}$ Interestingly, acute administration of PRX at a dose that resulted in clinically relevant serum concentrations did not affect enhanced freezing. Wang et al. have also demonstrated the efficacy of PRX on PTSD-like symptoms in a modified SPS model. ${ }^{[59]}$ In their study, a single electric footshock was given to rats immediately after administration of SPS (SPS + Shock). PRX administered for 14 days immediately after SPS+Shock prevented the induction of PTSD-like symptoms such as enhanced conditioning fear responses and anxiety behaviors. Taken together, the effect of PRX on PTSD-like symptoms in SPS rats suggests a sufficient degree of predictive validity for this animal model. Further studies are needed to evaluate the efficacy of other types of SSRIs and drugs, such as propranolol, on PTSD-like symptoms in SPS rats.

In addition to the criteria mentioned above, Yehuda and Antelman previously proposed criteria specific for animal models of PTSD. ${ }^{[60]}$ The main aim of the criteria is to define the stressor in detail. As described above, SPS consists of three stresses: $2 \mathrm{hr}$ restraint, forced swim, and ether anesthesia, which correspond to psychological, physiological, and endocrinological stress, respectively. The sequence of stresses in the SPS model is somewhat arbitrary and does not simulate the common set of trauma experienced by patients with PTSD (i.e. lack of ecological validity). However, each of the three stresses markedly increase serum corticosterone levels, and by combining the three different stresses, the SPS model could achieve severity of symptoms similar to that of PTSD. Also, the risk of habituation processes that diminish the effect of the stressor could be avoided. Furthermore, SPS can lead to enhanced negative feedback of the HPA axis 7 days after SPS, through TDS, and this endocrinological characteristic, which has been consistently replicated in the SPS model, is one of the advantages of SPS over other animal models of PTSD.

One of the limitations of the SPS model, according to the criteria by Yehuda and Antelman, is that the intensity of the stressor cannot be modified; the stressor does not produce PTSD-like symptoms in a dosedependent manner. It is unclear whether or not SPS can induce bi-directional emotional responses; reduced responsiveness to the stressor (avoidance and/or numbing) has not been well studied. Lastly, further studies are needed to determine the degree of inter-individual variability in response to stressors in the SPS model.

\section{CONCLUSIONS}

The behavioral and endocrinological responses to stimuli, molecular changes in the hippocampus, and therapeutic response to PRX and DCS observed in SPS rats support the face, construct, and predictive validity of this model as an animal analog of PTSD. The usefulness of this model is further supported by the interesting data generated by additional neurotransmitter studies and specific neurocircuitry studies. Overall, the findings show that the SPS paradigm has been partially validated as an animal model of PTSD. However, additional issues need to be addressed in order to refine and further validate the model; further studies are therefore warranted.

Acknowledgments. This work was supported by a grant-in-aid for general scientific research from the Ministry of Education, Science, and Culture of Japan, a Health Science Research Grant for Research on Brain Science from the Ministry of Health and Welfare of Japan, and a grant from Core Research for Evolutional Science and Technology (CREST) of Japan Science and Technology (JST).

\section{REFERENCES}

1. American Psychiatric Association. Diagnostic and Statistical Manual of Mental Disorders. 4th ed. Washington, DC: American Psychiatric Association; 1994.

2. Kessler RC, Sonnega A, Bromet E, et al. Posttraumatic stress disorder in the National Comorbidity Survey. Arch Gen Psychiatry 1995;52:1048-1060.

3. Orr SP, Pitman RK, Lasko NB, et al. Psychophysiological assessment of posttraumatic stress disorder imagery in World War II and Korean combat veterans. J Abnorm Psychol 1993;102: 152-159.

4. Liberzon I, Krstov M, Young EA. Stress-restress: effects on ACTH and fast feedback. Psychoneuroendocrinology 1997;22: $443-453$.

5. Liberzon I, Lopez JF, Flagel SB, et al. Differential regulation of hippocampal glucocorticoid receptors mRNA and fast feedback: relevance to post-traumatic stress disorder. J Neuroendocrinol 1999;11:11-17.

6. Harvey BH, Naciti C, Brand L, et al. Endocrine, cognitive and hippocampal/cortical $5 \mathrm{HT} 1 \mathrm{~A} / 2 \mathrm{~A}$ receptor changes evoked by a time-dependent sensitisation (TDS) stress model in rats. Brain Res 2003;983:97-107.

7. Wakizono T, Sawamura T, Shimizu K, et al. Stress vulnerabilities in an animal model of post-traumatic stress disorder. Physiol Behav 2007;90:687-695.

8. Shimizu K, Kikuchi A, Wakizono T, et al. Present status and prospects about an animal model of post-traumatic stress disorder in rats using a shuttle box. Nihon Shinkei Seishin Yakurigaku Zasshi 2006;26:155-168.

9. Siegmund A, Wotjak CT. Hyperarousal does not depend on trauma-related contextual memory in an animal model of posttraumatic stress disorder. Physiol Behav 2007;90:103-107.

10. Siegmund A, Wotjak CT. A mouse model of posttraumatic stress disorder that distinguishes between conditioned and sensitised fear. J Psychiatr Res 2007;41:848-860.

11. Rau V, DeCola JP, Fanselow MS. Stress-induced enhancement of fear learning: an animal model of posttraumatic stress disorder. Neurosci Biobehav Rev 2005;29:1207-1223.

12. Cohen H, Zohar J, Matar MA, et al. Setting apart the affected: the use of behavioral criteria in animal models of post traumatic stress disorder. Neuropsychopharmacology 2004;29:1962-1970. 
13. Adamec R, Muir C, Grimes M, et al. Involvement of noradrenergic and corticoid receptors in the consolidation of the lasting anxiogenic effects of predator stress. Behav Brain Res 2007;179: 192-207.

14. Adamec RE, Blundell J, Burton P. Relationship of the predatory attack experience to neural plasticity, pCREB expression and neuroendocrine response. Neurosci Biobehav Rev 2006;30:356-375.

15. Cohen H, Kaplan Z, Matar MA, et al. Long-lasting behavioral effects of juvenile trauma in an animal model of PTSD associated with a failure of the autonomic nervous system to recover. Eur Neuropsychopharmacol 2007;17:464-477.

16. Cohen H, Kaplan Z, Matar MA, et al. Anisomycin, a protein synthesis inhibitor, disrupts traumatic memory consolidation and attenuates posttraumatic stress response in rats. Biol Psychiatry 2006;60:767-776

17. Stam R. PTSD and stress sensitisation: a tale of brain and body. Part 2: animal models. Neurosci Biobehav Rev 2007;31:558-584.

18. Cohen H, Zohar J, Gidron Y, et al. Blunted HPA axis response to stress influences susceptibility to posttraumatic stress response in rats. Biol Psychiatry 2006;59:1208-1218.

19. Shimizu K, Sawamura T, Nibuya M, et al. An animal model of posttraumatic stress disorder and its validity: effect of paroxetine on a PTSD model in rats. Nihon Shinkei Seishin Yakurigaku Zasshi 2004;24:283-290.

20. Yehuda R, Southwick SM, Krystal JH, et al. Enhanced suppression of cortisol following dexamethasone administration in posttraumatic stress disorder. Am J Psychiatry 1993;150: 83-86.

21. Yehuda R. Biology of posttraumatic stress disorder. J Clin Psychiatry 2001;62:41-46.

22. Yehuda R, Golier JA, Halligan SL, et al. The ACTH response to dexamethasone in PTSD. Am J Psychiatry 2004;161:1397-1403.

23. Yehuda R, Boisoneau D, Lowy MT, et al. Dose-response changes in plasma cortisol and lymphocyte glucocorticoid receptors following dexamethasone administration in combat veterans with and without posttraumatic stress disorder. Arch Gen Psychiatry 1995;52:583-593.

24. Sapolsky RM, Armanini MP, Packan DR, et al. Glucocorticoid feedback inhibition of adrenocorticotropic hormone secretagogue release. Relationship to corticosteroid receptor occupancy in various limbic sites. Neuroendocrinology 1990;51:328-336.

25. Chambers RA, Bremner JD, Moghaddam B, et al. Glutamate and post-traumatic stress disorder: toward a psychobiology of dissociation. Semin Clin Neuropsychiatry 1999;4:274-281.

26. Harvey BH, Oosthuizen F, Brand L, et al. Stress-restress evokes sustained iNOS activity and altered GABA levels and NMDA receptors in rat hippocampus. Psychopharmacology (Berl) 2004;175: 494-502.

27. Heresco-Levy U, Javitt DC. The role of $N$-methyl-D-aspartate (NMDA) receptor-mediated neurotransmission in the pathophysiology and therapeutics of psychiatric syndromes. Eur Neuropsychopharmacol 1998;8:141-152.

28. Yamamoto S, Morinobu S, Fuchikami M, et al. Effects of single prolonged stress and D-cycloserine on contextual fear extinction and hippocampal NMDA receptor expression in a rat model of PTSD. Neuropsychopharmacology 2008;33:2108-2116.

29. Shiah IS, Yatham LN. GABA function in mood disorders: an update and critical review. Life Sci 1998;63:1289-1303.

30. Geuze E, van Berckel BN, Lammertsma AA, et al. Reduced GABAA benzodiazepine receptor binding in veterans with posttraumatic stress disorder. Mol Psychiatry 2008;13:74-83, 73.

31. Khan S, Liberzon I. Topiramate attenuates exaggerated acoustic startle in an animal model of PTSD. Psychopharmacology (Berl) 2004;172:225-229.
32. Orr SP, Metzger LJ, Lasko NB, et al. Physiologic responses to sudden, loud tones in monozygotic twins discordant for combat exposure: association with posttraumatic stress disorder. Arch Gen Psychiatry 2003;60:283-288.

33. Pitman RK, Orr SP, Shalev AY, et al. Psychophysiological alterations in post-traumatic stress disorder. Semin Clin Neuropsychiatry 1999;4:234-241.

34. Iwamoto Y, Morinobu S, Takahashi T, et al. Single prolonged stress increases contextual freezing and the expression of glycine transporter 1 and vesicle-associated membrane protein $2 \mathrm{mRNA}$ in the hippocampus of rats. Prog Neuropsychopharmacol Biol Psychiatry 2007;31:642-651.

35. Kohda K, Harada K, Kato K, et al. Glucocorticoid receptor activation is involved in producing abnormal phenotypes of single-prolonged stress rats: a putative post-traumatic stress disorder model. Neuroscience 2007;148:22-33.

36. Takahashi T, Morinobu S, Iwamoto Y, et al. Effect of paroxetine on enhanced contextual fear induced by single prolonged stress in rats. Psychopharmacology (Berl) 2006;189:165-173.

37. Imanaka A, Morinobu S, Toki S, et al. Importance of early environment in the development of post-traumatic stress disorder-like behaviors. Behav Brain Res 2006;173:129-137.

38. Steru L, Chermat R, Thierry B, et al. The tail suspension test: a new method for screening antidepressants in mice. Psychopharmacology (Berl) 1985;85:367-370.

39. Chermat R, Thierry B, Mico JA, et al. Adaptation of the tail suspension test to the rat. J Pharmacol 1986;17:348-350.

40. Quirk GJ, Garcia R, Gonzalez-Lima F. Prefrontal mechanisms in extinction of conditioned fear. Biol Psychiatry 2006;60:337-343.

41. Rauch SL, Shin LM, Phelps EA. Neurocircuitry models of posttraumatic stress disorder and extinction: human neuroimaging research—past, present, and future. Biol Psychiatry 2006;60: 376-382.

42. Milad MR, Rauch SL, Pitman RK, et al. Fear extinction in rats: implications for human brain imaging and anxiety disorders. Biol Psychol 2006;73:61-71.

43. Rothbaum BO, Davis M. Applying learning principles to the treatment of post-trauma reactions. Ann N Y Acad Sci 2003;1008: $112-121$.

44. Milad MR, Orr SP, Lasko NB, et al. Presence and acquired origin of reduced recall for fear extinction in PTSD: results of a twin study. J Psychiatr Res 2008;42:515-520.

45. Heresco-Levy U, Kremer I, Javitt DC, et al. Pilot-controlled trial of D-cycloserine for the treatment of post-traumatic stress disorder. Int J Neuropsychopharmacol 2002;5:301-307.

46. Ressler KJ, Rothbaum BO, Tannenbaum L, et al. Cognitive enhancers as adjuncts to psychotherapy: use of D-cycloserine in phobic individuals to facilitate extinction of fear. Arch Gen Psychiatry 2004;61:1136-1144.

47. Hofmann SG. Enhancing exposure-based therapy from a translational research perspective. Behav Res Ther 2007;45:1987-2001.

48. Walker DL, Ressler KJ, Lu KT, et al. Facilitation of conditioned fear extinction by systemic administration or intra-amygdala infusions of D-cycloserine as assessed with fear-potentiated startle in rats. J Neurosci 2002;22:2343-2351.

49. Ledgerwood L, Richardson R, Cranney J. D-cycloserine and the facilitation of extinction of conditioned fear: consequences for reinstatement. Behav Neurosci 2004;118:505-513.

50. Bremner JD, Scott TM, Delaney RC, et al. Deficits in short-term memory in posttraumatic stress disorder. Am J Psychiatry 1993; 150:1015-1019.

51. Bremner JD, Randall P, Scott TM, et al. Deficits in short-term memory in adult survivors of childhood abuse. Psychiatry Res 1995;59:97-107. 
52. Bremner JD, Randall P, Scott TM, et al. MRI-based measurement of hippocampal volume in patients with combat-related posttraumatic stress disorder. Am J Psychiatry 1995;152:973-981.

53. Richter-Levin G. Acute and long-term behavioral correlates of underwater trauma-potential relevance to stress and post-stress syndromes. Psychiatry Res 1998;79:73-83.

54. Pitman RK, van der Kolk BA, Orr SP, et al. Naloxone-reversible analgesic response to combat-related stimuli in posttraumatic stress disorder. A pilot study. Arch Gen Psychiatry 1990;47: 541-544.

55. Willner P. Validation criteria for animal models of human mental disorders: learned helplessness as a paradigm case. Prog Neuropsychopharmacol Biol Psychiatry 1986;10:677-690.
56. Liberzon I, Sripada CS. The functional neuroanatomy of PTSD: a critical review. Prog Brain Res 2008;167:151-169.

57. Albucher RC, Liberzon I. Psychopharmacological treatment in PTSD: a critical review. J Psychiatr Res 2002;36:355-367.

58. Harvey BH, Naciti C, Brand L, et al. Serotonin and stress: protective or malevolent actions in the biobehavioral response to repeated trauma? Ann N Y Acad Sci 2004;1032:267-272.

59. Wang W, Liu Y, Zheng H, et al. A modified single-prolonged stress model for post-traumatic stress disorder. Neurosci Lett 2008;441:237-241.

60. Yehuda R, Antelman SM. Criteria for rationally evaluating animal models of posttraumatic stress disorder. Biol Psychiatry 1993;33:479-486. 